\title{
Vergiftungen in der Schweiz
}

\author{
Hugo Kupferschmidt ${ }^{a}$, Christine Rauber-Lüthy ${ }^{b}$ \\ a Dr. med., eMBA-HSG, Direktor Tox Info Suisse, Zürich; ${ }^{\text {b }}$ Dr. med., Stv. des Direktors und Leitende Ärztin
}

* Der Schweregradbewertung durch Tox Info Suisse liegt der Poisoning Severity Score zugrunde (Persson HE et al. Clin Toxicol 1998; 36: 205-13). Er beruht auf der Bewertung von Einzelsymptomen und -befunden nach festen Kriterien. Eine Vergiftung ist demnach leicht, wenn Symptome auftreten, die wenig beein trächtigend sind und in der Regel spontan wieder verschwinden. Eine mittelschwere Intoxikation liegt dann vor, wenn behandlungswürdige und länger anhaltende Symptome vorhanden sind. Schwere Symptome sind ausnahmslos immer behandlungswürdig, lebensbedrohlich, und/ oder führen zu bleibenden Beeinträchtigungen.

\begin{abstract}
Tox Info Suisse führte 201437427 Beratungen durch, knapp 34500 Beratungen zu Giftexpositionen und 3000 prophylaktischer Natur. Gut 16500 Giftexpositionen betrafen Kinder, 83\% davon jünger als 5 Jahre. Knapp drei Viertel aller Vergiftungen geschehen mit Medikamenten, Haushaltsprodukten oder Pflanzen. Fünf der zehn Todesfälle gehen auf das Konto der Medikamentenvergiftungen, zwei auf dasjenige von technisch-gewerblichen Chemikalien, je einer von Kosmetika und Pflanzen, und einer war die Folge von Drogenkonsum. Von den schweren Fällen sind 70\% durch Medikamente und 14\% durch Genussmittel und Drogen verursacht.
\end{abstract}

51,2\% der Expositionen betrafen Kinder, mehrheitlich im Vorschulalter (82,6\% aller Expositionen bei unter 16-Jährigen traten bei Kindern <5 Jahren auf). Bei der Geschlechtsverteilung war bei den Kindern ein leichtes Überwiegen der Knaben (51,2\% vs. 47,1\% Mädchen) und bei den Erwachsenen der Frauen (57,6\% vs. 41,9\% Männer) zu sehen. 88\% der knapp 24000 unbeabsichtigten (akzidentellen) Vergiftungen ereigneten sich im häuslichen Milieu, bei den knapp 4900 beabsichtigten Intoxikationen trat die grösste Anzahl (72\%) im Rahmen von Suizidversuchen auf.

\section{Schwere und tödliche Vergiftungen in der Schweiz 2014}

Von 193 schweren* Vergiftungen mit Medikamenten (davon 5 Kinder) ereigneten sich 161 mit Mitteln für das Nervensystem, im wesentlichen Analgetika (v.a. Opioide, Paracetamol), Antiepileptika (Phenobarbital, Carbamazepin, Lamotrigin, Valproinsäure und Levetiracetam) und Psychopharmaka (Benzodiazepine $n=30$, Antidepressiva $n=40$, Antipsychotika $n=34$, davon Quetiapin $n=16$, Zolpidem und Zopiclon $n=7$ ). Bei den

\section{Intoxications en Suisse}

En 2014, Tox Info Suisse a recensé 37427 demandes de renseignement dont près de 34500 avec exposition et environ 3000 de nature préventive. Plus de 16500 expositions concernaient des enfants, dont $83 \%$ âgés de moins de 5 ans. Près de trois quarts de toutes les intoxications sont dues aux médicaments, aux produits domestiques et aux plantes. Cinq cas fatals sur les dix recensés étaient des intoxications dues aux médicaments, deux aux produits chimiques industriels (I'un agricole, l'autre cosmétique) et un cas à des drogues. Parmi les cas graves, $70 \%$ ont été causés par des médicaments et $14 \%$ par des produits d'agrément et des drogues. übrigen schweren Medikamentenintoxikationen waren Präparate für den Gastrointestinaltrakt (Insulin $\mathrm{n}=3$, und Glimepirid $n=1$ ), für den Kreislauf (Digoxin $n=1$ ), für den Atmungstrakt (Antitussiva vom Opioidtyp $\mathrm{n}=1$, Diphenhydramin $\mathrm{n}=5$, Chlorpheniramin $\mathrm{n}=1$, Promethazin $\mathrm{n}=1$ ) und für den Bewegungsapparat (Mefenaminsäure $\mathrm{n}=7$, Tolperison $\mathrm{n}=2$, Tizanidin $\mathrm{n}=1$ ) beteiligt. Die restlichen schweren Vergiftungen wurden durch alkoholische Desinfektionsmittel $(n=4)$, Methotrexat $(n=2)$, Phenprocoumon $(n=1)$, Ciprofloxacin $(n=1)$, Ifosfamid $(n=1)$ und Scopolamin $(n=1)$ verursacht.

Bei den fünf Todesfällen durch Medikamente waren bei zwei in suizidaler Absicht Trimipramin, Lorazepam, Amitriptyline, Levomepromazine, Oxycodone, Paracetamol, Metamizol, Zolpidem und (in einem Fall) Ethanol in unterschiedlicher Kombination eingenommen worden. In einem Fall war eine versehentliche Einnahme von Flecainid und Bisoprolol die Ursache. Zweimal war Methotrexat die Ursache einer tödlichen Vergiftung, einmal, weil es täglich anstatt wöchentlich eingenommen worden war, und einmal zusammen mit einer Paracetamol-Überdosierung.

Genussmittel, Drogen und Alkohol führten zu 38 schweren und einer tödlichen Vergiftung. 20 betrafen Alkoholvergiftungen, acht Halluzinogene und Stimulanzien (inkl. Ecstasy), vier Kokain, und fünf Opiate. Einer war die Folge von Gammahydroxybutyrat (GHB). Dazu kam eine Vergiftung mit «Poppers» (flüchtige Nitrite). Bei den Alkoholvergiftungen wurden mit einer Ausnahme auch noch Medikamente oder andere Drogen konsumiert.

Bei den fünf schweren Opiatintoxikationen, vier davon mit Heroin, stand das typische klinische Bild mit Koma und Atemdepression im Vordergrund, obschon bei allen weitere Drogen mitkonsumiert worden waren (Alkohol, Kokain, Benzodiazepine, Cannabis). Alle Patienten erholten sich.

Bei den sieben Patienten, die Halluzinogene (LSD = Lysergsäurediethylamid) und Stimulanzien (z.B. Amphetamin, Methamphetamin, MDMA [Methylendio xymethamphetamin/«Ecstasy»], Phencyclidin [PCP], Methylendioxypyrovaleron [MDPV] und Mephedron), teils zusammen mit weiteren Drogen, konsumiert hatten, traten Agitation, Halluzinationen, aber auch Koma auf, dazu Hyperthermie und schwere Rhabdomyolysen. Vor allem unter den synthetischen Kathinonen kam es zu schwerster Agitation. Bei einer jungen Frau kam es 
nach Konsum von Speed zum Kammerflimmern und zum Tod.

Bei vier Patienten, alles Männer, die Kokain mit anderen Stimulanzien und Alkohol konsumiert hatten, kam es zu ausgeprägten sympathomimetischen Zeichen mit Agitation, Krampfanfällen, Stenokardien, neurologischen Ausfällen und Rhabdomyolyse.

Ein Patient machte nach Absetzen einen schweren GHBEntzug durch mit Rigor, Tremor, Schwitzen, Agitation und Desorientierung. Er musste sediert und intubiert werden. Bei einem anderen Mann kam es nach Einnahme von Poppers (flüchtige Nitrite) und Alkohol zu einer schweren Methämoglobinämie, die die Gabe des Antidots Methylenblau nötig machte. Nach vorübergehender Agitation war der Verlauf komplikationslos. Expositionen mit Pflanzen führten 2013 zu drei schweren und einer tödlichen Vergiftung. Einer dieser Fälle betraf ein dreiwöchiges Kind, das wegen Verdauungsbeschwerden Sternanistee (Illicium verum) erhielt und daraufhin Krampfanfälle entwickelte; durch den Nachweis von Anisatin in den Früchten wurde der Nachweis erbracht, dass der Tee mit dem toxischen japanischen Anis (Illicium anisatum) kontaminiert war. Ein männlicher Patient trank einen Tee aus Engelstrompete (Brugmansia aut Datura suaveolens), entwickelte ein schweres anticholinerges Syndrom mit Koma, Tachykardie, Mydriasis und trockenen Schleimhäuten, wurde mit Physostigmin behandelt und erholte sich innert eines Tages vollständig. Eine Frau erlitt nach dem Genuss von Bärlauch, der durch Verwechslung mit Herbstzeitlosenblättern (Colchicum autumnale) vermischt war, eine schwere Colchicinvergiftung mit Leukopenie, Thrombopenie und Agranulozytose, Niereninsuffizienz und Leberschädigung. Sie erholte sich unter konservativer Therapie innert drei Wochen. Ein Mann entwickelte nach der Einnahme von Eisenhutwurzeln (Aconitum napellus) schwere Kammerarrhythmien, an denen er trotz
Gabe von Lipidemulsion und intensivmedizinischen Massnahmen verstarb.

Bei den Haushaltsprodukten traten 15 schwere Intoxikationen auf, darunter drei bei Kleinkindern. Bei zwei dieser Kinder kam es durch Aspiration nach Trinken von niedrigviskösen Kohlenwasserstoffen (Petrol bzw. Anzündflüssigkeit) zu Pneumonien, und ein drittes Kind erlitt Verätzungen des oberen Gastrointestinaltraktes nach akzidenteller Einnahme eines salpetersäurehaltigen Kalk- und Rostlösers. Ein Erwachsener verschluckte Lampenöl zusammen mit einem Entkalker und Medikamenten, wurde bewusstlos, musste erbrechen und erlitt schwere Koagulationsnekrosen vom Oesophagus bis ins Duodenum ohne Perforation. Er musste intubiert werden und entwickelte septische Komplikationen, erholte sich aber nach Tagen. Ein anderer Mann entwickelte ein Alveolarödem mit Ateminsuffizienz nach Einnahme und Aspiration eines Fleckenschutzsprays, der niedrigvisköse Kohlenwasserstoffe enthält; er erholte sich nach wenigen Tagen. Eine grosse Menge Grillanzündflüssigkeit zusammen mit Alkohol und Medikamenten trank ein junger Mann, was zu einem Koma führte. Ebenfalls ein tiefes Koma war die Folge der Einnahme von Ethanol (Brennsprit bzw. Händedesinfektionsmittel) bei zwei Männern mittleren Alters. Eine betagte Frau erlitt ausgedehnte Ulzerationen und Nekrosestrassen im gesamten oberen Gastrointestinaltrakt nach der Einnahme einer grösseren Menge handelsüblichen Entkalkers. Sie erholte sich unter konservativer Behandlung innert einiger Tage. Die Einnahme grösserer Mengen ethylenglykolhaltiger Kühlerflüssigkeit fürs Auto bewirkte bei einer jungen Frau zweimal eine ZNS-Depression und eine metabolische Azidose; sie wurde beide Male hämodialysiert und erholte sich ohne Entwicklung einer Niereninsuffizienz. Ein Mann mittleren Alters trank einen Reiniger für Geschirrspülmaschinen, aspirierte und entwickelte eine metabolische Azidose und schwere pul-

Tabelle 1: Häufigkeit der Vergiftungen beim Menschen nach Noxengruppen (Tox Info Suisse 2014).

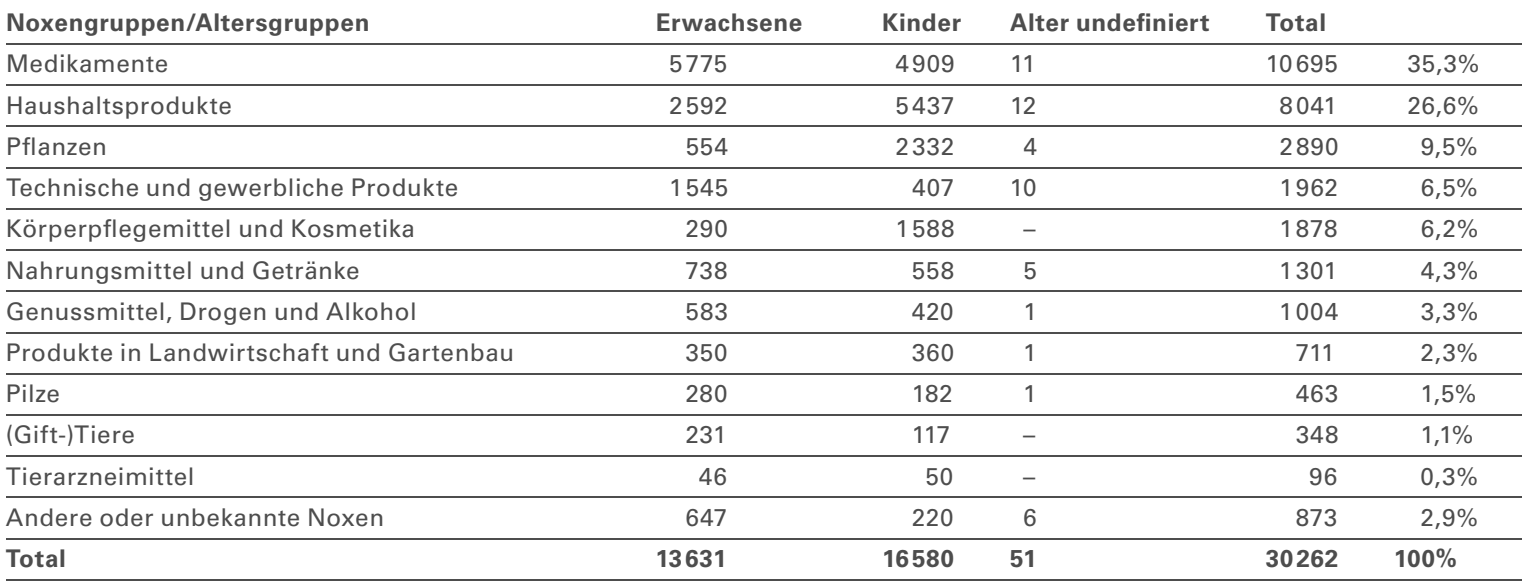


Tabelle 2: Häufigkeit der Noxengruppen und Vergiftungsschweregrad der auswertbaren ärztlichen Rückmeldungen (STIZ 2013) zu Giftkontakt beim Menschen (nur hohe Kausalität), Medikamente nach ATC-Codegruppen.

\begin{tabular}{|c|c|c|c|c|c|c|c|c|c|c|c|c|}
\hline \multirow[b]{2}{*}{$\begin{array}{l}\text { Noxengruppen/ } \\
\text { Schweregrad }\end{array}$} & \multicolumn{3}{|c|}{ Erwachsene } & \multicolumn{5}{|c|}{ Kinder } & \multicolumn{4}{|c|}{ Total } \\
\hline & 0 & $\mathbf{L}$ & M & $\mathbf{S}$ & $\mathbf{T}$ & 0 & $\mathbf{L}$ & M & S & $\mathbf{T}$ & & \\
\hline Medikamente & 371 & 1341 & 470 & 188 & 5 & 317 & 220 & 61 & 5 & - & 2978 & $62,6 \%$ \\
\hline \multicolumn{13}{|l|}{ davon } \\
\hline Nervensystem & 245 & 1109 & 360 & 157 & 2 & 97 & 126 & 39 & 4 & - & 2139 & \\
\hline Atemwege & 14 & 55 & 23 & 8 & - & 47 & 24 & 7 & - & - & 178 & \\
\hline Bewegungsapparat & 39 & 71 & 30 & 9 & - & 38 & 17 & 4 & 1 & - & 209 & \\
\hline Kreislauf & 27 & 39 & 17 & 1 & 1 & 36 & 9 & 1 & - & - & 131 & \\
\hline Verdauung & 7 & 14 & 14 & 14 & - & 29 & 13 & 4 & - & & 85 & \\
\hline übrige & 39 & 53 & 26 & 9 & 2 & 70 & 31 & 6 & & & 236 & \\
\hline Haushaltsprodukte & 37 & 147 & 31 & 12 & - & 102 & 125 & 14 & 3 & - & 471 & $9,9 \%$ \\
\hline Technische und gewerbliche Produkte & 35 & 273 & 52 & 11 & 2 & 10 & 40 & 7 & 1 & - & 431 & $9,1 \%$ \\
\hline Genussmittel, Drogen und Alkohol & 21 & 120 & 136 & 38 & 1 & 10 & 18 & 6 & - & - & 350 & $7,4 \%$ \\
\hline Pflanzen & 9 & 22 & 26 & 2 & 1 & 28 & 30 & 7 & 1 & - & 126 & $2,6 \%$ \\
\hline Pilze & 2 & 29 & 14 & 2 & - & 24 & 3 & 1 & - & - & 52 & $1,1 \%$ \\
\hline Körperpflegemittel und Kosmetika & 7 & 21 & 1 & - & 1 & 22 & 36 & 2 & - & - & 90 & $1,9 \%$ \\
\hline Produkte für Landwirtschaft und Gartenbau & 4 & 23 & 8 & 4 & - & 7 & 4 & 1 & - & - & 51 & $1,1 \%$ \\
\hline (Gift-)Tiere & 4 & 14 & 8 & 5 & - & 3 & 10 & 7 & 1 & - & 52 & $1,1 \%$ \\
\hline Nahrungsmittel und Getränke & 4 & 10 & 9 & - & - & 4 & 6 & 2 & - & - & 35 & $0,7 \%$ \\
\hline Tierarzneimittel & 3 & 1 & - & 1 & - & 1 & 2 & - & - & - & 8 & $0,2 \%$ \\
\hline Andere oder unbekannte Noxen & 6 & 47 & 17 & 4 & - & 5 & 10 & 1 & - & - & 90 & $1,9 \%$ \\
\hline Total & 503 & 2048 & 772 & 267 & 10 & 533 & 504 & 109 & 11 & - & 4757 & $100 \%$ \\
\hline
\end{tabular}

monale Komplikationen mit mehrmonatiger Hospitalisation und Rehabilitation. Ein anderer Mann musste wegen Verätzungen und Schwellung im Rachenbereich nach Verschlucken eines natronlaugehaltigen Rohrreinigers notfallmässig intubiert werden. Eine Frau erbrach Frischblut nach Einnahme von Nagellack, des Inhalts eines HotCold-Packs und einer Glasscherbe.

Mit Kosmetika und Körperpflegemitteln waren 2014 ein tödlicher und drei mittelschwere Vergiftungen bei zwei Kindern und zwei Erwachsenen zu verzeichnen. Zwei mittelschwere Fälle betrafen zwei gut einjährige Kleinkinder, die auf dem Wickeltisch die Babypuderdose erwischten und den talkumhaltigen Inhalt über sich schütteten. Sie entwickelten in der Folge einen starken anhaltenden Husten, der eine medizinische Überwachung nötig machte. Die beiden Erwachsenen tranken beide eine grosse Menge Flüssigseife bzw. Shampoo, was zu einer Pneumonie führte, an welcher einer der beiden Patienten verstarb.

Mit Nahrungsmitteln und Getränken kam es $2014 \mathrm{zu}$ keiner schweren, wohl aber neun mittelschweren Vergiftungen, die durch unverträgliche oder verdorbene Nahrungsmittel, Meeresfrüchte, Chilischoten und (koffeinhaltige) Nahrungsergänzungsmittel bedingt waren. Ein halbwüchsiger Knabe versuchte, einen Gegenstand mit dem Mund aus Mehl zu fischen, worauf er aspirierte und Husten und Atemnot entwickelte.
Zu zwölf schweren und zwei tödlichen Vergiftungen kam es durch technisch-gewerbliche Produkte, mit einer Ausnahme alle bei Erwachsenen. Ein Mann verstarb nach Einnahme von Zyankali, ein anderer überlebte eine ähnliche Episode. Zum anderen Todesfall kam es bei einem Erwachsenen nach Einnahme von ca. $300 \mathrm{~g}$ Natriumhydroxidpellets für die Abflussreinigung; er verstarb an den Folgen von Oesophagusverätzungen und einer Magenperforation. Jedes Jahr führen Ätzstoffe zu schweren Vergiftungen: Ein eineinhalbjähriges Kleinkind erwischte zuhause 80\%ige Essigsäure, die in der Küche verwendet wird, und erlitt durch die Einnahme schwere Verätzungen des Rachens und der Speiseröhre. Es musste wegen der schwellungsbedingten Verlegung der Atemwege einige Tage intubiert werden und blieb vier Wochen hospitalisiert. Zu Verätzungen des oberen Gastrointestinaltraktes kam es bei einem Mann durch die Einnahme eines stark alkalischen Zementmörtelhärters, bei einem anderen durch basischen Melkmaschinenreiniger; beide überstanden die schweren inneren Verletzungen. Auch durch Inhalation von ätzenden Dämpfen kann es zu schweren Symptomen kommen: Einem Mann geriet am Arbeitsplatz ein Produkt aus fast reiner Essigsäure und Formaldehyd auf die Haut und in die Augen. Neben den kutanen Läsionen führte die Inhalation der Dämpfe zu Verätzungen der Atemwege, was die Intubation nötig 
machte. Er erlitt einen komplizierten Verlauf mit ARDS und mehrwöchiger Hospitalisation. Chlorhaltige Verbindungen können durch Säurebeimischungen Chlorgas freisetzen, das stark reizend auf die Atemwege wirkt. Am Arbeitsplatz waren dies bei einem Patienten Natriumhypochlorit und Phosphorsäure, bei einem zweiten eine chlorhaltige Schwimmbadchemikalie und Salzsäure, was zu Augenbrennen, starkem Husten und Atemnot führte. Beide erholten sich ohne Notwendigkeit der Intubation komplikationslos innert Tagen. Alkoholhaltige Chemikalien wie Flächen- oder Händedesinfektionsmittel können Anlass zu schweren Alkoholvergiftungen geben. Eine Frau wurde kurz nach der Einnahme verwirrt, agitiert und aggressiv, später tief komatös und musste intubiert werden. Eine andere Patientin wurde ebenfalls rasch komatös, erbrach blutig und aspirierte. Auch sie musste intubiert werden und machte einen komplizierten Verlauf durch. Eine andere Frau trank eine Kupfersulfatlösung, was rezidivierendes Erbrechen und vorübergehende Geschmacksstörung zur Folge hatte. Ein Mann entwickelte eine schwerste Methämoglobinämie, Hämolyse, Rhabdomyolyse durch grossflächige kutane Exposition mit reinem Anilin und überlebte dies trotz wiederholter Gabe des Antidots Methylenblau nur knapp. Eine junge Frau hantierte über längere Zeit mit elementarem Quecksilber, was massiv erhöhte Quecksilberblutspiegel $(1200 \mathrm{nmol} / \mathrm{L}$, normal <50) zur Folge hatte; sie wurde wiederholt mit DMPS cheliert und blieb längere Zeit hospitalisiert.

Mit Stoffen in Landwirtschaft und Gartenbau ereigneten sich vier schwere Vergiftungsfälle. Ein junger Mann wurde auf einem Zuckerrübenfermenter ohnmächtig und stürzte hinein. Es bestand der Verdacht auf silogasbedingte Kohlendioxidvergiftung ( $\mathrm{pCO}^{2} 68 \mathrm{~mm} \mathrm{Hg}$ ). Er musste wegen eines Kreislauf- und Atemstillstandes kurz reanimiert werden und zeigte danach eine ausgeprägte respiratorische Azidose, eine Hypothermie und die Zeichen der bronchialen Aspiration. Er wurde für zwei Tage intubiert, benötigte kurz Katecholamine und erhielt eine antibiotische Behandlung, mit rascher Erholung. Ein weiterer junger Mann stürzte in die Jauchegrube eines Schweinestalles und zog sich dabei neben einer Rippenkontusion asphyktische Beschwerden mit Agitation, Verwirrung, Desorientiertheit und Amnesie zu. Zudem musste er erbrechen. Radiologisch zeigte sich ein akut toxisch bedingtes alveoläres Lungenödem. Alle Symptome bildeten sich innert weniger Tage unter konservativer Behandlung zurück. Eine betagte Frau entwickelte nach Einnahme eines Flüssigdüngers und eines glyphosathaltigen Herbizids eine Hyperkaliämie von 7,2 mmol/l und eine metabolische Azidose. Sie wurde mit Bikarbonat und Insulin-Glu- kose behandelt und erholte sich rasch. Ebenfalls Pflanzendünger nahm ein älterer Mann ein; bei ihm trat eine schwere nitratbedingte Methämoglobinämie (60\%) auf, die erfolgreich mit dem Antidot Methylenblau behoben wurde. In einem letzten Fall wurde ein Mann Opfer einer Vergiftung durch Dritte mit dem Insektizid Imidacloprid. Er entwickelte Somnolenz und Erbrechen, eine schwere Rhabdomyolyse und einen Leberzellschaden, wovon er sich unter intensivmedizinischer Behandlung wieder erholte.

Giftige Tiere: Insgesamt kam es 2014 zu 15 mittelschweren und sechs schweren Bissen oder Stichen durch giftige Tiere (16 Schlangen*, ein Fisch, eine Qualle, eine Spinne und zwei Insekten), sieben bzw. einer davon bei Kindern.

Pilze: Im letzten Jahr ereigneten sich zwei schwere Pilzvergiftungen. In beiden, von einander unabhängigen, Fällen ass ein Ehepaar selbstgesammelte, nicht kontrollierte Pilze, und beide Male entwickelten die Eheleute eine Gastroenteritis, und die beiden Frauen eine schwere Leberschädigung, von der sich beide unter intensivmedizinischer Betreuung erholten. Beide zeigten stark erhöhte Transaminasen und INR, aber keine Enzephalopathie. Beide wurden antidotal mit Silibinin und N-Acetylcystein behandelt. Im ersten Fall konnte der Pilzexperte in den Essensresten Amanita phalloides nachweisen, auch der Urintest auf Amanitin war deutlich positiv. Im zweiten Fall, bei dem sich die Patientin erst fünf Tage nach dem Genuss der Pilze vorstellte, war kein Pilzmaterial mehr vorhanden, und für den Nachweis im Urin war es zu spät. Hier führte die Beschreibung des Sammelgutes zur Verdachtsdiagnose. Bei beiden Ehemännern verlief die Vergiftung symptomatisch, aber milder.

Andere Noxen: Ein junger Mann entwickelte nach Rauchgasinhalation bei einem Hausbrand ein toxisches Lungenödem, aber keine CO-Vergiftung. Der Verlauf war durch eine gleichzeitig eingenommene Medikamentenüberdosis verkompliziert. Ein älterer Mann erlitt, ebenfalls bei einem Wohnungsbrand, eine Rauchgasvergiftung mit Koma und erhöhtem $\mathrm{COHb}(36 \%)$ und Laktat. Die Kohlenmonoxidintoxikation wurde mit Sauerstoff behandelt, bei Verdacht auf Zyanidvergiftung wurde Hydroxocobalamin gegeben. Damit erholte sich der Patient rasch. Bei einer älteren Frau führte ein schlecht ziehendes Cheminée im Schlaf zu einer Rauchgasexposition mit Kohlenmonoxidvergiftung (COHb 14\%) und toxischem Lungenödem. Sie erhielt Sauerstoff $100 \%$ und erholte sich unter intensivmedizinischer Behandlung. Auch der vierte Patient war bei einem Wohnungsbrand Rauchgasen ausgesetzt. Er war initial bewusstlos und sein $\mathrm{COHb}$ betrug 22,7\%. Er wurde mit Sauerstoff behandelt und erholte sich schnell. 